\title{
The development and process evaluation of the ACTION study: A person-centred communication intervention targeting nurse assistants in home care for older persons
}

\section{Tanja Gustafsson ( $\nabla$ tanja.gustafsson@hb.se)}

Hogskolan i Boras Akademin for vard arbetsliv och valfard https://orcid.org/0000-0002-8379-7910

Annelie J Sundler

University of Borås

Elisabeth Lindberg

University of Borås

Pernilla Karlsson

University of Borås

Hanna Maurin Söderholm

University of Borås

\section{Research article}

Keywords: Communication, Intervention, Education, Home care, Nurse assistants, Older persons, Personcentred

Posted Date: December 2nd, 2019

DOI: https://doi.org/10.21203/rs.2.17948/v1

License: (c) (i) This work is licensed under a Creative Commons Attribution 4.0 International License.

Read Full License 


\section{Abstract}

Background A rapidly ageing population challenges the health care system in general and home care services in particular. Communication is a cornerstone of person-centred care. However, little research has been conducted on how to improve communication between health care professionals and older persons in home care contexts, despite research showing the importance of such interactions. The increasing demands on how to best and efficiently improve competence in health professionals is the reason why the ACTION intervention was conducted. This paper aims to describe the development and process evaluation of an educational intervention for nurse assistants (NAs) in home care and highlights the potential of self-directed web-based learning as well as the pitfalls of conducting complex interventions in home care.

Methods A web-based educational intervention focusing on person-centred communication was developed that targeted NAs in home care for older persons. Twenty-seven NAs from two units in Sweden were recruited, and 23 NAs were offered the educational intervention. Data were collected from multiple sources before, during and after the intervention and were analysed using quantitative and qualitative methods. Data were extracted from the web platform and analysed to determine the NAs' engagement in the intervention. Additionally, interviews, evaluation forms and field notes were used to evaluate the feasibility of the intervention.

Results The main findings suggest that web-based education seems to be an appropriate strategy in the home care context. The majority of the NAs (91\%) participated in full or in part in the intervention. During the implementation process, some adaptions were required to fit the local circumstances regarding technical support, scheduling, and the design of the lectures. The NAs perceived the format to be easy to use and flexible and appreciated the stepwise modules. The content was perceived as valuable.

Conclusions Our findings show that the benefits of the web-based educational intervention included the short and focused lectures as well as its accessibility. Challenges with the implementation process included gaining access to the NAs and motivating and involving the NAs. This study emphasizes the environmental support needed to successfully conduct complex interventions, including physical, organizational and cultural aspects.

\section{Background}

This study focused on home care services for older persons; it aimed to develop and evaluate an educational intervention for person-centred communication targeted at nurse assistants (NA). Home care services are important to support better ageing for older persons at home. Today, there is a strong emphasis on person-centred care. The provision of such care needs to be based on communication and interaction between NAs and older persons and include respect, dignity, and the acknowledgement of individual needs. Interaction and communication with older persons is important (1). Thus, communication is a cornerstone promoting person-centred care (PCC). However, there may be 
communication barriers that need to be acknowledged to facilitate effective and person-centred communication with older persons $(2,3)$. A greater focus on communication and how to improve the communication competency of NAs in home care is necessary (4).

\section{Home care for older persons}

There are reasons to believe that home care services will be even more important in the future due to alterations to healthcare policies and the demographics of the rapidly ageing population (5). People in late old age are at risk of adverse health outcomes and may need home care services to remain living at home. Moreover, home care visits may be meaningful as a social activity and can contribute to preserving dignity and humanity, particularly for those living alone. However, being in need of home care also means being forced to adapt to routines and relations, not by choice but because they are assigned by someone else $(3,6)$. Previous work has indicated that older persons' individual needs are often vaguely expressed and may be difficult to detect (3). Responding to older persons' physical, emotional and social needs may be challenging and requires the communicative competence of professionals.

In addition to the increasing needs for home care services and the challenges of providing them, the literature points to a competence gap (7). A relatively large proportion of employees in home care are reported to have low qualification levels and some staff lack sufficient competency to ensure that older persons receive the care they require $(5,7)$. In Sweden, where this study was conducted, there are two types of home care services: nursing and social care performed by NAs, such as help with daily living or personal care and hygiene, and home healthcare service performed by registered nurses, such as drug administration. This study focuses on nursing and social care performed by NAs for older persons in their own homes.

Home care of older persons is embedded in everyday conversations, where small talk can be used to elicit information, normalise unpleasant procedures and manage interactions and relationships (8). Hence, communication and interaction is a cornerstone of the achievement of nursing goals. Understanding and showing sensitivity to older persons' expressions is necessary to respond to individual needs and enhance PCC (4). In addition, the dignity and humanity of older persons can be preserved through sensitive listening and individualized care (9). Previous research has identified challenges related to the communication with and care of older persons in their homes $(1,4,10,11)$. Such challenges are related to older persons' fragility, existential concerns, and emotional needs (10). Furthermore, the needs of older persons may be vaguely expressed and difficult to detect $(4,10,11)$. This might risk individuals being misinterpreted or ignored. There may also be difficulties related to organizational structures $(1,10)$, for instance, strict rules and regulations for home care visits (1). In addition to these difficulties, communication failure can lead to problems, such as older persons' feelings of insecurity or increased feelings of loneliness (12). 


\section{Theoretical underpinnings of the person-centred communication intervention}

The specific situations of persons in need of care, who are often fragile, require an understanding of not only their physical and practical needs but also the existential aspects of a changing life situation (13). The caring science perspective underscores the importance of considering the patient to be an entity and his or her world to be the focus of caring. A dialogue in which the patients' vulnerability, hope and wishes are included is a prerequisite for a caring relationship (14). This perspective is in line with PCC, which emphasizes the individual who is in need of care and that person's rights. Moreover, the use of reflection is important in practice development with a focus on PCC. Such practice development must be meaningful and engage those who are participating in the process (15).

PCC is widely recognized as a key concept related to quality of care and is defined as care in which "individuals' values and preferences are elicited and guide all aspects of health care, supporting their realistic health and life goals" (16, p. 16). The theoretical foundation of PCC is the theory and philosophy of Carl Rogers (17), which emphasizes a caring approach based on the principles and values of acceptance, caring, empathy and sensitivity in human interactions. This is in line with current literature on PCC, which stresses communication, respect, autonomy, empathy and empowerment as key components $(16,18,19)$. PCC refers to a caring approach that is based on humanistic values and individual needs and that emphasizes patients being treated as persons. Thus, PCC involves a caring approach that is respectful and compassionate, as well as support for patients' capacity for autonomy (20). The approach emphasizes mutual respect and understanding (21). An ability to be sensitive and listen is essential, as is the ability to provide acknowledgement and confirmation of emotions (22). Consequently, PCC can be accomplished through and supported by the development of communication competences.

In addition to PCC framework, the work of Watzlawick et al. (23) provides a basis for understanding communication. These researchers developed an understanding of how and why human communication and interactions take place and stated that interaction always involves communication. Everything one does is a message; actions, words and silences all convey a message. Furthermore, all communication is related to "a content and a relationship aspect" (23, p. 54), as the communication of content and interaction are interwoven. There have been a limited number of studies on communication practices in home care services. Further research on how to improve the communication skills of NAs in this setting is needed.

\section{Barriers to and facilitators of the improvement of person-centred communication in home care}

Recently, there has been an increased focus on promoting PCC. Considering the communicative and relational nature of PCC, PCC also influences caregivers. PCC may have a positive impact on job satisfaction (24), but further studies on this topic are required. Moreover, NAs need support and education 
to develop the knowledge, skills and attitudes required to meet the individual needs of older persons (25). Working in home care services is characterized by mobile, dynamic and sometimes unpredictable working conditions. NAs move between home care units and the homes of older persons according to a combination of set schedules and the emergence of care needs. This poses challenges for NAs' competence development and learning. Previous work $(26,27,28,29)$ has proposed flexible learning approaches such as web-based or blended approaches that combine e-learning and face-to-face interactions. These types of approaches have been effective in a wide range of health professional contexts and purposes, e.g., for providing advice and support to cancer survivors (28), conducting motivational interviewing (27), and changing clinician behaviour (26). A meta-analysis of 201 articles performed by Cook et al. (29) described positive effects of web-based training for health care professionals, compared to no intervention, on knowledge outcomes, skills, learner behaviour and patient effects. Research on how to best and most efficiently improve PCC and communication competency in home care contexts needs further exploration.

\section{Methods}

\section{Aim}

The aim of this paper was to describe the development and process evaluation of the ACTION intervention. The following research questions were addressed:

1. What were the NAs levels of adherence to the intervention?

2. What were the NAs expectations for the intervention?

3. What were the NAs experiences of the implementation of the intervention?

4. What experiences were described by the NAs following the intervention?

\section{Design and development of the intervention}

In the ACTION study, a web-based educational intervention for person-centred communication targeted at NAs in home care for older persons was developed. This paper reports the development and process evaluation of the intervention. During the development of the intervention, previously implemented complex interventions in applied clinical research were considered with the aim of succeeding in applying an effective and feasible intervention (30). A team consisting of researchers and teachers with expertise in the area developed the intervention. During the planning and development of the intervention, users and stakeholders in home care were involved.

The intervention was designed to enhance learners' communication competency through flexible webbased education that combined theoretical knowledge with reflections, supervision and course leader 
feedback. The backbone of the intervention was a web-based learning platform where several types of content and learning strategies were combined, including video lectures, movie clips, quizzes and reflection assignments, which were complemented with in-person group sessions with supervision. The content was structured in eight stepwise modules (see Table 1) focusing on communication and PCC from a caring science perspective; the content was intended to be distributed over eight weeks, with approximately one module administered each week. Two of the modules were organized as live group sessions to be conducted at the workplace. The first four modules were pre-recorded when developing the intervention. The last three modules were designed based on the participants input and requests regarding the content and structure and consequently, were recorded during the early stages of the intervention.

Table 1. Overview of the content of the eight educational modules. 


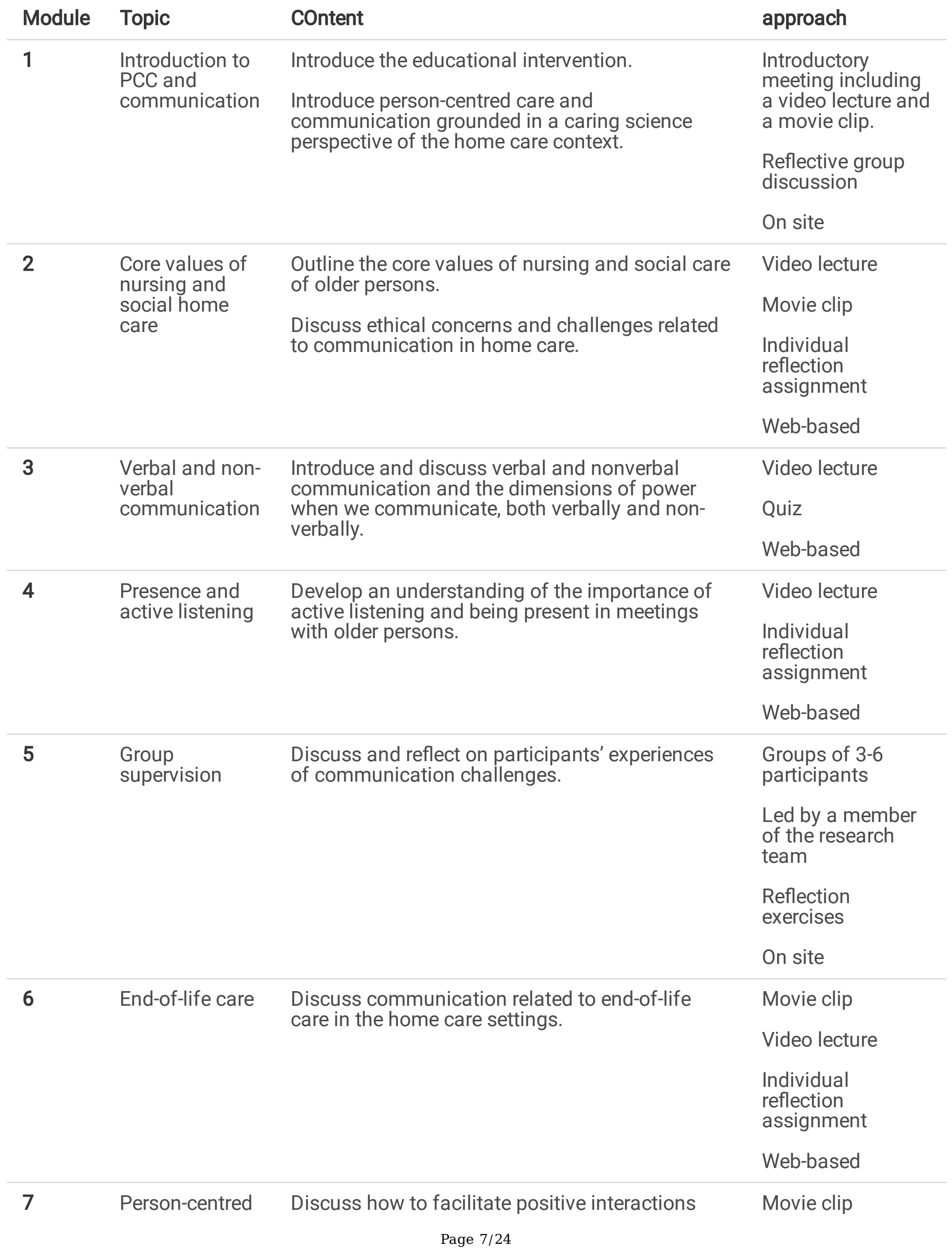


and emotional conversations and person-centred communication, including in conversations about existential matters with older persons.
Video lecture

Quiz

Web-based

Video lecture

Individual

reflection

assignment

Course leader

feedback

Web-based

To provide flexibility, the NAs themselves decided when to carry out each module. Each module was planned to take a maximum of 15-30 minutes, and the movie clips and web-based lectures used were between 5-25 minutes in length. The quiz or reflection assignment for the current module had to be completed before the NA could proceed to the next module. The intention of the assignments was to enhance self-directed learning and to support deep learning and reflections and prevent the participants from "clicking through" the course pages without engaging with the content. The educational content was tailored to NAs and built upon the previously described framework of person-centred care and communication with older persons, in addition to previous research from the COMHOME project (31).

\section{Study setting and recruitment procedures}

The study was planned and prepared in 2017, and the intervention was performed in spring 2018. A convenience sample of NAs was derived from a middle-sized community in western Sweden. The eligibility criterion for participants was having a permanent job position enabling engagement in the intervention and data collection. All eligible NAs from two geographical districts at the home care service organization were recruited, resulting in 27 NAs. Of these NAs, one declined to participate, one was on long-term sick leave, and three terminated their employment. After one new employee was hired, 23 NAs were eventually offered the intervention. The research team planned how the NAs would participate in the educational intervention, but the manager of the home care units, the team leaders and the NAs themselves were responsible for organizing the time and resources for the intervention at their home care units. For an overview of the study process, see Figure 1.

Figure 1. Flow chart of the ACTION study process, including the recruitment of participants and data collection. 


\section{Data collection}

Data were collected from multiple sources before, during and after the intervention, and consisted of user analytics data and data from pre- and post-interviews, evaluation forms, and field notes (see Figure 1).

Before the start of the intervention, data were collected via participant demographic questions, group interviews and field notes. Twenty-two of the NAs participated in four group interviews, which focused on their expectations and worries regarding the upcoming educational intervention. Towards the end of these group interviews, the NAs were asked to write personal notes about their thoughts on anonymous Post-lt notes.

During the course of the intervention, user data were extracted from the web-based platform on the NAs individual engagement. These logs included the time (hh:mm:ss) spent on each module, number of visits, and whether the participant had completed all parts of each module.

After the intervention, NAs evaluated the educational intervention, regardless of their level of engagement, by submitting an evaluation form. The form consisted of four questions on NAs' experiences of the design and content of the intervention. Repeated reminders were sent by e-mail and distributed at visits, and ultimately, fifteen evaluation forms were received. In addition, a purposive sample of five participants participated in individual interviews, to provide insight on the NAs' experiences of the intervention. The interviews were semi-structured and all started with the introductory question, "How did you experience the education?", with follow-up questions such as "What do you mean?" and "Can you tell me more?". The interviews lasted from 30-60 minutes.

Field notes were written continuously during the process; the contents of field notes included reports from visits; notes from discussions with management, team leaders and NAs; reflections; e-mail communication; and questions to discuss with the project team. The field notes were used to gather input on the process, design and content of the intervention.

\section{Data analysis}

User statistics from the web-based platform were analysed using IBM SPSS Statistics version 25 to analyse the participant flow and identify patterns in adherence and use. To analyse participants' adherence and activity on the web-based system, time and number of visits in each module were analysed. Pearson correlation tests were performed to analyse the potential impact of background variables, e.g., age, gender, and language, on adherence. Interviews, evaluation forms and field notes were analysed qualitatively to explore the NAs' experiences of the intervention. A qualitative content analysis by Elo and Kyngäs (32) was performed. In this step, the data were read several times and then coded and grouped, resulting in three categories: expectations and worries before the intervention; the implementation process, and experiences from the intervention. In the results, quotations from the interviews and evaluation forms were used to illuminate the NAs' experiences. The analysis was mainly 
performed by two members of the research team, and further discussed and validated by all authors. All authors read and commented on the final version of the analysis to ensure the rigour of the categories described.

\section{Results}

The results are presented in five sections: (I) demographic characteristics of the participants, (II) participants' adherence to the intervention, (III) expectations and worries before the intervention, (IV) the implementation process, and $(\mathrm{V})$ experiences from the intervention.

\section{Demographic characteristics of the participants}

The demographic characteristics of the 23 NAs who were offered the educational intervention are presented in Table 2. The group had a median age of 39. The gender distribution ( male=3, female=20) was in line with the current overall characteristics of NAs, as the NA profession is female dominated. NAs 'years of experiences working in home care varied. One-third $(n=7)$ of the participants had a native language other than Swedish.

Table 2. Demographic characteristics of the participants. 
$\mathrm{N}=23$

Age in years

MD

39

Range

24-61

Gender

Female, $\mathrm{n}$

20

Male, $\mathrm{n}$

3

Employment

Full-time (100\%), n 5

Part-time (60-90\%), n 13

Missing, $\mathrm{n} \quad 5$

Home care work experience in years

Median

Range

7

$1-40$

Current workplace tenure in years

Median

4

Range

0-18

Native language

Swedish, $\mathrm{n}$

16

Other, $\mathrm{n}$

7

\section{Participants' adherence to the intervention}

The majority of the NAs had participated to a large extent in the educational intervention (see Figure 2). Most NAs, $91 \%(n=21)$, completely or partially participated in five or more modules. A few of the NAs, $9 \%$ $(n=2)$, participated in four or fewer modules. After the fifth module, some of the participants dropped out. The last module was completed by half of the NAs $(n=12,52 \%)$.

Figure 2. Overview of the participants' adherence to the eight modules of the education. 
No correlations were found between participation and background variables such as age, work experience, gender or education. There were positive correlations (r-values ranging from .421-.666, $p<0.05$ ) between the number of visits on each page and whether the participant had a native language other than Swedish. Non-native speakers showed more than twice the amount of activity in terms of number of visits and spent more time on each module than participants who were native Swedish speakers.

\section{- Expectations and worries before the intervention}

Overall, the NAs' expectations before the education included both curiosity and concern. Not knowing what was to come was exciting and led to interest in what the content could contribute to their knowledge and competence development.

"It's still exciting, to see what this leads to and what one can benefit from it."

Another desire the NAs had for the educational intervention was to receive confirmation on the appropriateness of their current communication with the older persons they met.

"Because I don't know if I do it right. Or, to comfort [someone], that can be very hard sometimes. Am I doing it right or wrong?"

The NAs expressed concern regarding the potential time constraints of completing the education modules and management of the time needed for the education modules during their busy working hours.

"How would we get the time needed for this? That is something to think about. But, if this is something we will do, then time needs to be allocated, I guess. Yes. But I don't know how."

At this point, the NAs expressed that the information they had received on the design and content of the educational intervention were limited. They did not express any concern regarding the web-based format, and they looked forward to being introduced and beginning it.

\section{The implementation process}

Important aspects during the implementation of the intervention were found to be related to reminders, time constraints, when to actually complete the educational intervention, the equipment at the workplaces, and modification of the lectures. 
The web-based intervention consisted of weekly modules. Each week, a newsletter was sent by e-mail with concise information about the forthcoming module. This reminder was posted on a bulletin board in the staff room. Throughout the intervention, one member of the research team, the first author, acted as the NAs' primary point of contact and visited their workplaces on several occasions. In addition, another member of the research team also performed repeated visits to support and evaluate the implementation.

During the first weeks of the intervention, the NAs expressed problems with time constraints. The problem was discussed with their manager and additional time to complete the intervention was allotted in their schedules. However, the time the NAs took to complete the modules competed with their other administrative tasks, making it challenging to complete everything. Problems managing the time needed for each module every week continued, even though the manager decided to bring on additional staff during the intervention. Particularly during periods of high workload, it seemed to be a struggle to prioritize completion of the intervention, for example, when acute situations arose in the workplace and the NAs had to help their colleagues, resulting in interruption of the modules. To allow some additional time for the NAs to catch up if needed, halfway into the intervention, the time limit for each module was extended from one to two weeks.

Questions also arose regarding the physical location where the NAs were expected to work with the course material. The manager's recommendation was to primarily do this at the workplace during their working hours. If necessary, e.g., due to a tight schedule or heavy workload, completion of the modules from home and being compensated for this as over-time was allowed. This resulted in some NAs preferring to undertake the education at home to not be disturbed.

There were benefits with the web-based format being flexible for NAs and allowing the research team to follow their progression during the education. The web-based format allowed for early detection of those who fell behind or did not follow the modules according to the plan. These NAs were contacted through email or during one of the visits by the researcher, and they were offered help if there were any technical or other problems. Some difficulties in contacting NAs arose, as some did not respond to e-mails or rarely were at the workplace during visits. The team leaders helped contact those individuals. A few NAs mentioned technical problems logging on to the web platform. After they contacted the responsible researcher, help was received from the IT support at the university.

Another challenge noted was the limited number of desktop computers, with two per home care unit, allocated for the intervention and their everyday work. Some NAs expressed worries concerning noise and privacy when listening to the web-based lectures and when writing assignments. To establish a sense of 
empowerment and secure resource allocation, the research team decided to provide the home care units with tablets and headphones.

During the implementation of the intervention, some NAs indicated that the first lectures were too long and disengaging in some parts. This feedback was taken into account when developing the last three modules, resulting in shorter lectures and more engaging approaches, such as conversation-based lectures including several presenters.

\section{Experiences from the intervention}

Overall, the NAs found web-based education to be easy to use, and appreciated the stepwise modules. Some NAs found the web format challenging due to a lack of digital experience.

"Too much using the computer, especially when you're not that technical."

Compared to traditional education, the web-based education format included benefits such as accessibility and flexibility to independently decide when to undergo the modules. The tablets and headphones offered were appreciated by the NAs, as they allowed for additional flexibility and privacy.

"I think that it [the education] was great! It was great that you could log in and then that it was stepwise. // That you could sit down and do it whenever you wanted. It gives you independence. You don't have to be there at a specific moment // It gives you more independence. I think that it was very positive!"

"I have done them [the modules] on the tablet. Very good! // I just needed to sit down for a while and shut everything out. It was great!"

The overall structure and variation was appreciated, but some NAs expressed that the intervention consisted of too many modules. Another weakness with the intervention being web-based was the lack of interaction with the lecturers, and some NAs asked for more engaging forms of lectures. Some lectures perceived as too long, resulting in a loss of interest or the module being interrupted.

"I think that the education was lengthy, too many modules. Maybe it would have been more interesting if it was shortened a bit."

"Maybe it could be more... not just someone standing there and reading, maybe more pictures or examples or something. That would be interesting, if something more happened."

The group discussion was reported to be one of the most appreciated parts of the intervention. This discussion allowed the NAs to reflect with colleagues on different aspects of communication. The content became more meaningful and interesting. 
"I think that... when we had these four parts [the first four modules] and then this group session... it was... you need to meet, so to say. I think that it's good that some parts are at the computer, but you also need to meet each other. Because then you feel that... you meet and have a dialogue. So that it's not just... it becomes routine, so to say... I like meeting the others."

"It was great, the group session. It was interesting. It gave me a lot. Yes. I think everybody thought so. It was great; that is something we said afterwards."

The opportunity to discuss difficult matters with each other was valuable and provided the NAs with useful insights into their daily work. There was a desire for more opportunities similar to the group discussion and to meet in groups and to reflect together on different aspects of home care. Others thought that one session was sufficient.

The short movie clips were found to be particularly interesting and stimulating. These could provide nuance and deepen the participants' understandings of the education content.

"Some of the movie clips have been good. I remember one in particular that was about end-of-life care. The nurse assistants in the movie did it all so beautifully, and it consisted of the most important parts."

All the modules except the group supervision were followed by either a quiz or a reflection assignment. The NAs reported that the quizzes were fun and that the reflection assignments were meaningful parts of their learning but that they were time consuming. Participants completing the reflection assignment in the final module received written feedback from the researchers. This feedback was expressed as positive and valuable since it provided confirmation on how the NAs reflected on their current communication ability.

"I think that the reflective assignments gives you more... They feel better... but they are more burdensome and time consuming. /... / The first two weeks, I felt that this is something I must do at home so you can think more; you need more time for them."

Some NAs perceived the intervention as burdensome, and a few expressed that they felt forced to participate. They also expressed frustration with the lack of involvement in the decision phase and felt that the intervention imposed an additional workload, which influenced their motivation to complete the modules. Other aspects that reduced the NAs' motivation during the intervention were vacations, sick leaves, and other prioritized training.

"This [the intervention] is something that the manager has agreed to and then we have to accept that. End of discussion."

"You can't say that just because it is boring, you don't do it. There is no excuse for it. It is a part of your duty. Yes. Everything is not fun, that is how I feel." 
However, the NAs reported that the content was relevant and interesting and that it provided valuable knowledge that would be useful in their daily work. NAs with extensive work experience in home care settings expressed already having sufficient knowledge. They felt that parts of the content were repetitive, but they valued the opportunity to reflect on their everyday work practice. Some NAs also believed that the intervention was more suitable for NAs without previous work experience.

"Yes, I think it [the intervention] is useful. It actually relates to what we do all the time at work. Very useful. /.../ So, it was needed. Even if you already have some awareness of it, it's always needed ... to get more knowledge or to think about it even more."

"I think that the education was great. Above all, for those with limited experience in healthcare, but not that many new insights for me, as I have been working for more than twenty years."

\section{Discussion}

To our knowledge, this is the first study to use a web-based approach to improve person-centred communication skills among nurse assistants in home care. The intervention seems to be an appropriate strategy in this context compared to traditional classroom- or group-based education, but further improvements are required. Our findings emphasize the benefits of the web-based learning format, the challenges of implementation particular to the home care context, and the importance of organizational support and resources.

\section{Web-based learning in home care settings}

The feasibility of the ACTION intervention was confirmed by the relatively high level of adherence among the NAs and because it was generally well received with respect to both format and content. The main advantage of the intervention was the web-based format, with learning modules designed to be short and focused. The course content was easy to access and required limited time commitment from the NAs. Today, there is an increased demand for e-learning in nursing overall (26), especially in the area of communication $(25,33)$. Evidence suggests that web-based education on communication can improve healthcare professionals' communication self-efficacy (34), which in turn is a strong predictor for learning and future performance (35). While our results are in line with those of previous work considering timeand cost-effectiveness for both trainers and trainees (36), there are challenges in balancing this approach with requests for group discussions and face-to-face lectures, which are more time intensive and less flexible. Furthermore, the content could be developed, e.g., by including demonstrations of real-world situations and, as suggested by Bravender et al. (37), interactive components, to further motivate participants and support their learning of communication skills. Overall, this approach shows potential for providing education to a broad population of health care professionals for relatively little cost, while longer, more in-depth training may jeopardize feasibility. Additional benefits include the design flexibility in terms of possibilities to continuously adapt and make changes to both the format and content according to local circumstances and participant feedback, which is in line with the MRC guidance (30) for the implementation of complex interventions. 


\section{Implementation challenges}

During the implementation process, challenges with gaining access to the NAs arose. Initially, the manager facilitated researchers' full access to the NAs at the home care units. In practice, access was difficult, mostly due to the NAs' working conditions. During the onsite visits by the researchers, contact was primarily made with the team leaders and occasionally with the manager but seldom with the NAs. Most of the information and support offered by the researchers was addressed to the team leaders, making them informal local facilitators who were important in supporting the implementation and participant involvement. Consequently, the importance of local facilitators was underestimated. As emphasized by Schön et al. (37) and Rogers (38), the importance of local champions when implementing innovative tools and technologies in organizations must be addressed. Hence, as this study shows, identifying, planning for and clarifying the different roles of those involved in the intervention, and the functions and expectations related to these roles, is crucial early in the process. Thus, teamwork with local stakeholders, facilitators and participants is important to succeed in the implementation of clinical interventions.

\section{Organizational support and resources}

When participating in this type of self-directed learning intervention, high levels of participant involvement, motivation and responsibility are required for successful implementation. Hence, both the physical environment, e.g., equipment and locations, as well as the workplace culture need to be supportive to enhance NAs' participation in the intervention. This is often referred to as facilitating conditions and has been identified as a crucial factor for the successful implementation of new technologies and tools $(38,39)$. In this study, the computers at the home care units were insufficient and were therefore completed with headphones and tablets. This improvement was not only necessary but also appreciated by the NAs. Another practical problem that emerged in the initial phase of the implementation was the lack of time assigned for the NAs. The education was something that the NAs were supposed to engage in independently "when they found the time to do so" during their heavy workdays. This approach failed almost immediately, resulting in specifically allocated time for the intervention. The participants expressed frustration regarding their lack of involvement in the initial decision phase.

During the early phases of the implementation, attempts were made to involve the NAs in the planning of the content, and suggestions were collected and implemented in the final modules. The NAs' frustration indicates the important role of management regarding attitudes and commitment not only in the decision phase but also during the whole process to retain the motivation needed to complete the education. This finding is in line with those of Venkatesh and Bala (39), who emphasized the importance of managerial efforts both pre- and post-implementation, as well as those of MacCormack, Dewing and McCance (21), who described the importance of creating a culture that can sustain person-centred care in both health care teams and organizations when developing PCC. To effect such changes, these authors underlined 
that organizations cannot rely on the motivation of individual participants. Instead, commitment is required from all levels to support active learning and achieve a potential culture change.

\section{Strengths and limitations}

Conducting, implementing and evaluating complex interventions is challenging, as emphasized by Craig et al. (30). This small-scale study attempted to address some of these challenges in the context of home care. Although analysis of learning outcomes or effects of the intervention was not included in this study, the findings show some promise with respect to how this type of intervention can be conducted. Nevertheless, a number of limitations need to be recognized, including the number of included participants, interpretation of the data, and involvement of researchers during the process.

The number of participants and the specifics of the local context in this study may limit its generalizability and impact, as in any small-scale intervention. Nonetheless, as recommended by the MRC guidelines (30), these types of studies are important since they examine key uncertainties identified during development and implementation. The experiences reported from this intervention contribute knowledge of the factors that appear to influence the applicability and use of this type of intervention.

In this study, we chose a mixed-method approach. Qualitative data from interviews, evaluation forms and field notes gave valuable information on the implementation process and the NAs' experiences of the intervention, while quantitative user analytics data contributed to the understanding of the NAs' engagement with the course content. Both methods have their benefits and their limitations with respect

to data collection and interpretation: the data from the web-based platform showed a high degree of participation, and the details of every NA's activity in each module (e.g., time spent and clicks) could be extracted. However, care should be taken when interpreting these variables in terms of learning outcomes because, as in any indirect observation, it is impossible to know the exact reasons behind the amount of time spent or actions taken at a particular course module page. As such, the post-interviews provided valuable complementary insights. However, there were some potential limitations regarding the data collection. Throughout the intervention, one member of the research team (the first author), had the main responsibility for the implementation and evaluation processes (e.g., evaluation forms and interviews) and was also responsible for developing some of the course modules. This involvement generated both benefits, in terms of establishing trust, and risks, e.g., whether participants felt that they could be honest during interviews. To address this issue, the evaluation also included anonymous written individual evaluation forms. As reported, these forms included both positive and negative feedback.

\section{Conclusions}

This process evaluation identifies important challenges and considerations for the successful conduct of complex interventions in home care settings. We found that the benefits of the web-based format were the short and focused lectures, as well as its accessibility and efficiency. Implementing the intervention in 
the home care context was challenging, due to the NAs' working conditions. Gaining access to the NAs and motivating and involving the NAs were identified as important aspects of the implementation process. This study emphasizes the importance of a supportive environment, including physical, organizational and cultural surroundings, when conducting a complex intervention in a home care setting. If designed and implemented appropriately, web-based self-directed approaches such as the one studied in this paper can be useful tools in the quality improvement of home care services. Similar educational interventions can be developed for not only nurse assistants but also other health care professionals and for different care contexts.

\section{Abbreviations}

PCC: person-centred care; NA: nurse assistant.

\section{Declarations}

\section{Ethics approval and consent to participate}

The study has been approved by the regional ethical review board in Gothenburg (Dnr. 260/17) and conforms to the Declaration of Helsinki. Written and verbal information about the study was given to all participants, and informed consent was obtained. The sampling of participants and data storage, flow and access are outlined in accordance with legislation and safety routines to safeguard the security, privacy and confidentiality of participants. Handling of personal information is accordance with the General Data Protection Regulation (GDPR).

\section{Consent for publication}

Not applicable.

\section{Availability of data and materials}

The dataset generated and analysed during the current study is available from the corresponding author on reasonable request.

\section{Competing interests}

The authors declare that they have no competing interests.

\section{Funding}

This research was funded by the Faculty of Caring Science, Work Life and Social Welfare at the University of Borås, and the Agneta Prytz-Folke och Gösta Folke foundation. The sponsors had no influence on or any other involvement in the study.

\section{Authors' contributions}


AJS initiated the study. The study design was proposed by AJS and developed by EL and HMS. Data collection was performed by TG and PK, and analysis was performed and discussed among all participating researchers. TG, AJS and HMS drafted the manuscript, and all authors read and commented on the manuscript and approved the final draft.

\section{Acknowledgements}

We sincerely thank the study participants for their time and effort during the intervention. Furthermore, we wish to thank the management for facilitating the intervention and for letting us take part in the NAs' daily work.

\section{Author information}

Tanja GUSTAFSSON, RN, MScN, Faculty of Caring Science, Work Life and Social Welfare, University of Borås, Sweden, tanja.gustafsson@hb.se

Annelie J SUNDLER, RN, Associate Professor, Faculty of Caring Science, Work Life and Social Welfare, University of Borås, Sweden, annelie.sundler@hb.se

Elisabeth LINDBERG, RN, PhD, Senior Lecturer, Faculty of Caring Science, Work Life and Social Welfare, University of Borås, Sweden, elisabeth.lindberg@hb.se

Pernilla KARLSSON, RN, MScN, Lecturer, Faculty of Caring Science, Work Life and Social Welfare, University of Borås, Sweden, pernilla.karlsson@hb.se

Hanna MAURIN SÖDERHOLM, PhD, Senior Lecturer, Faculty of Librarianship, Information, Learning and IT; PreHospen Centre for Prehospital Research; Faculty of Caring Science, Work Life and Social Welfare, University of Borås, Sweden, hanna.maurin@hb.se

\section{References}

1. Choe K, Kim K, Lee K-S. Ethical concerns of visiting nurses caring for older people in the community. Nurs Ethics. 2015;22(6):700-710.

2. Ruan J, Lambert VA. Differences in perceived communication barriers among nurses and elderly patients in China. Nurs Health Sci. 2008;10(2):110-6.

3. Öresland S, Määttä S, Norberg A, Jörgensen MW, Lutzen K. Nurses as guests or professionals in home health care. Nurs Ethics. 2008;15(3):371-83.

4. Sundler AJ, Eide H, van Dulmen S, Holmström IK. Communicative challenges in the home care of older persons - a qualitative exploration. J Adv Nurs. 2016;72(10):2435-44.

1. Thomé B, Dykes AK, Hallberg IR. Home care with regard to definition, care recipients, content and outcome: systematic literature review. J Clin Nurs. 2013;12(6):860-72. 
6. Jarling A, Rydström I, Ernsth-Bravell M, Nyström M, Dalheim-Englund AC. Becoming a guest in your own home: Home care in Sweden from the perspective of older people with multimorbidities. Int $J$ Older People Nurs. 2018;13(3):e12194.

7. Bing-Jonsson PC, Foss C, Bjørk IT. The competence gap in community care: Imbalance between expected and actual nursing staff competence. Nordic J Nurs Science. 2016;36(1):27-37.

8. Macdonald LM. Expertise in Everyday Nurse-Patient Conversations: The Importance of Small Talk. Glob Qual Nurs Res. 2016;11;3:2333393616643201.

9. Anderberg P, Lepp M, Berglund A-L, Segesten K. Preserving dignity in caring for older adults: a concept analysis. J Adv Nurs. 2007;59(6):635-43.

10. Sundler AJ, Höglander J, Eklund JH, Eide H, Holmström IK. Older persons' expressions of emotional cues and concerns during home care visits. Application of the Verona coding definitions of emotional sequences (VR-CoDES) in home care. Patient Educ Couns. 2017;100(2):276-82.

11. Hafskjold L, Eide T, Holmström IK, Sundling V, van Dulmen S, Eide H. Older persons' worries expressed during home care visits: exploring the content of cues and concerns identified by the Verona coding definitions of emotional sequence. Patient Educ Couns. 2016;99:1955-1963.

12. Svanström R, Sundler AJ, Berglund M, Westin L. Suffering caused by care - elderly patients' experiences in community care. Int J Qual Stud Health Well-being. 2013;8: 20603

13. Udo C. The concept and relevance of existential issues in nursing. Eur J Oncol Nurs. 2014;18(4):34754.

14. Arman M, Ranheim A, Rydenlund K, Rytterström P, Rehnsfeldt A. The Nordic Tradition of Caring Science: The Works of Three Theorists. Nurs Sci Q. 2015;28(4):288-96.

15. Smith K. Reflection and person-centredness in practice development. Int Prac Develop J 2016;6(1).

16. American Geriatrics Society Expert Panel on Person-Centered C. Person-Centered Care: A Definition and Essential Elements. J Am Geriatr Soc. 2016;64(1):15-8.

17. Rogers CR. On becoming a person. New York: Houghton Mifflin Company; 1989/1995.

18. Sharma T, Bamford M, Dodman D. Person-centred care: an overview of reviews. Contemp Nurse. 2015;51:107-120.

19. Edvardsson D, Winblad B, Sandman PO. Person-centred care of people with severe Alzheimer's disease: current status and ways forward. Lancet Neuro. 2008;7(4):362-7.

20. Entwistle VA, Watt IS. Treating patients as persons: a capabilities approach to support delivery of person-centered care. Am J Bioeth. 2013;13(8):29-39.

21. McCormack B, Dewing J, McCance T. Developing person-centred care: Addressing contextual challenges through practice development. The Online Journal of Issues in Nursing. 2011;16(2):3.

22. McCabe C. Nurse-patient communication: an exploration of patients' experiences. J Clin Nurs. 2004;13(1):41-9.

23. Watzlawick P, Beavin JH, Jackson DD. Pragmatics of Human Communication. New York: W.W. Norton \& Company; 1967. 
24. van den Pol-Grevelink A, Jukema JS, Smits CH. Person-centred care and job satisfaction of caregivers in nursing homes: a systematic review of the impact of different forms of person-centred care on various dimensions of job satisfaction. Int J Geriatr Psychiatry. 2012;27(3):219-29.

25. Lyttle DJ, Ryan A. Factors influencing older patients' participation in care: a review of the literature. Int J Older People Nurs. 2010;5(4):274-82.

26. Sinclair PM, Kable A, Levett-Jones T, Booth D. The effectiveness of Internet-based e-learning on clinician behaviour and patient outcomes: A systematic review. Int J Nurs Stud. 2016;57:70-81.

27. Fontaine G, Cossette S, Heppell S, Boyer L, Mailhot T, Simard M-J, et al. Evaluation of a Web-Based ELearning Platform for Brief Motivational Interviewing by Nurses in Cardiovascular Care: A Pilot Study. J Med Internet Res. 2016;18(8):e224.

28. Murphy, J, Worswick, L, Pulman, A, Ford, G, Jeffery, J. Translating research into practice: Evaluation of an e-learning resource for health care professionals to provide nutrition advice and support for cancer survivors. Nurs Educ Today. 2015;35(1), 271-6.

29. Cook DA, Levinson AJ, Garside S, Dupras DM, Erwin PJ, Montori VM. Internet-based learning in the health professions: a meta-analysis. JAMA. 2008;300(10):1181-96.

30. Craig P, Dieppe P, Macintyre S, Michie S, Nazareth I, Petticrew M. Medical Research Council Guidance. Developing and evaluating complex interventions: the new Medical Research Council guidance. 2008;337:a1655.

31. Hafskjold L, Sundler AJ, Holmström IK, Sundling V, Van Dulmen S, Eide, H. A cross-sectional study on person-centred communication in the care of older people: the COMHOME study protocol. BMJ Open. 2015;5(4):e007864.

32. Elo S, Kyngäs H. The qualitative content analysis process. J Adv Nurs. 2008;62(1):107-15.

33. Tulsky JA. Interventions to enhance communication among patients, providers, and families. J Palliat Med. 2005;8 Suppl 1:S95-102.

34. Caris-Verhallen W, Kerkstra A, Bensing JM, Grypdonck MH. Effects of video interaction analysis training on nurse patient communication in the care of the elderly. Patient Educ Couns. 2000;39(1):91-103.

35. Banduro A. Self-efficacy: The exercise of control. Basingstoke: W.H. Freeman; 1997.

36. Bravender T, Tulsky JA, Farrell D, Alexander SC, Ostbye T, Lyna P, et al. Teen CHAT: Development and utilization of a web-based intervention to improve physician communication with adolescents about healthy weight. Patient Educ Couns. 2013;93(3):525-31.

37. Schön UK, Grim K, Wallin L, Rosenberg D, Svedberg P. Psychiatric service staff perceptions of implementing a shared decision-making tool: a process evaluation study. Int J Qual Stud Health Wellbeing. 2018;13(1):1421352.

38. Rogers, E. M. Diffusion of innovations. Simon and Schuster; 2010.

39. Venkatesh $\mathrm{V}$, Bala $\mathrm{H}$. Technology acceptance model 3 and a research agenda on interventions. Decision Sci. 2008;39(2), 273-315. 
Figures

$\begin{aligned} & \text { PHASE 1: Development and } \\ & \text { recruitment }\end{aligned}$
$\begin{aligned} & \text { - Development of the } \\ & \text { web-based educational } \\ & \text { modules on person- } \\ & \text { centred communication } \\ & \text { - Recruitment of NAs at } \\ & \text { two home care units } \\ & \text { - Information provision at } \\ & \text { workplace meetings }\end{aligned}$
- Data were collected via
- Field notes

\section{Figure 1}

Flow chart of the ACTION study process, including the recruitment of participants and data collection. 


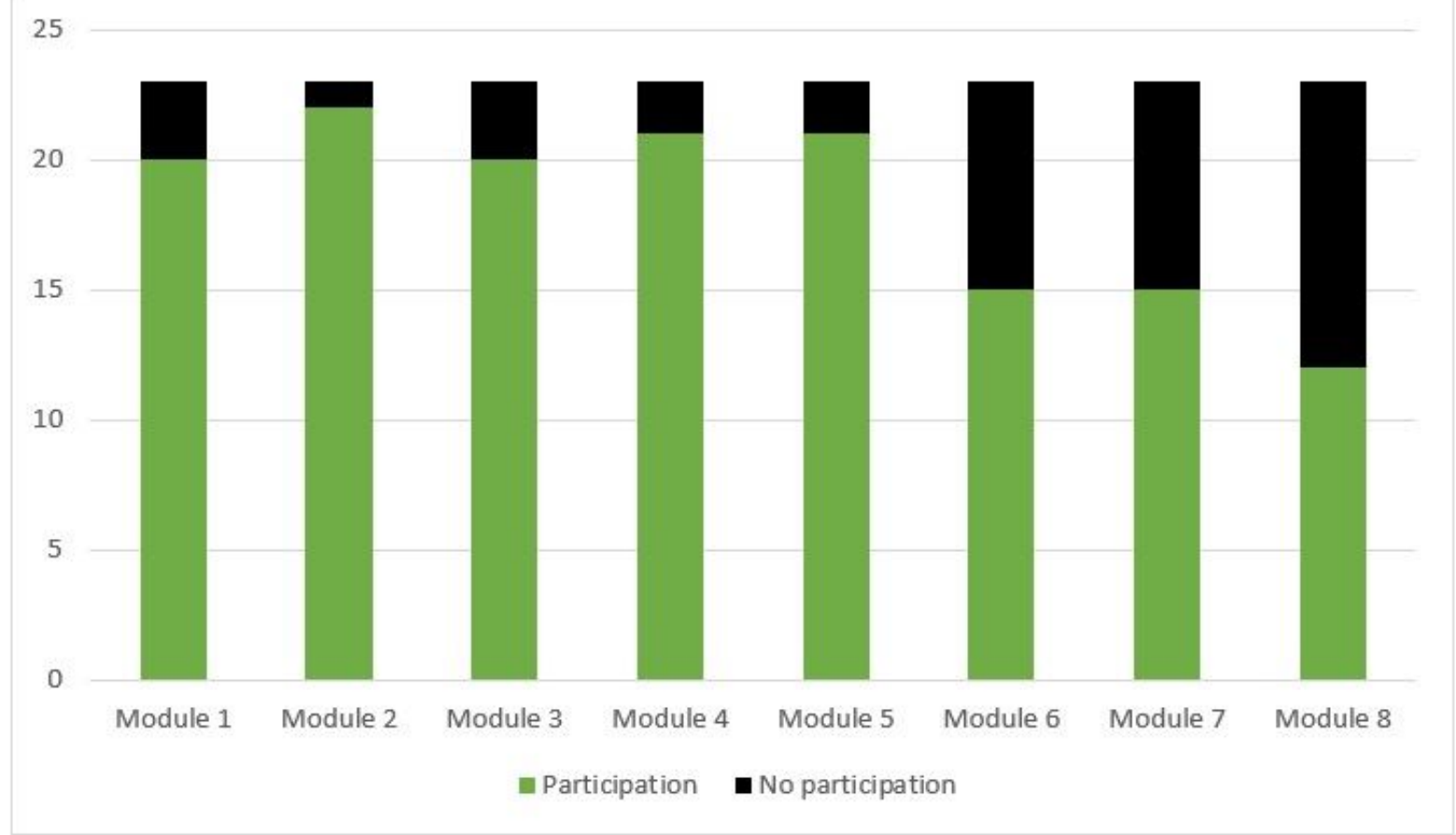

Figure 2

Overview of the participants' adherence to the eight modules of the education. 